\title{
Use of X-ray mutagenesis to increase genetic diversity of Zan- tedeschia aethiopica for early flowering, improved tolerance to bacterial soft rot and higher yield
}

\author{
Noam Reznik ${ }^{1 *}$, Bijaya Sharma Subedi ${ }^{1,2}$, Shoshana Weisman ${ }^{3}$, Gavriel Friesem ${ }^{1}$, Nir Carmi ${ }^{4}$, Iris Yedidia ${ }^{1, *}$ and \\ Michal Sharon-Cohen ${ }^{5}$ \\ 1. Department of Ornamental Plants and Agricultural Biotechnology, The Volcani Center, P.O. Box 15159, \\ Rishon LeZion 7505101, Israel; noamr@volcani.agri.gov.il (N.R.); bijaya.subedi@mail.huji.ac.il (B.S.S.); sho- \\ shw@shaham.moag.gov.il (S.W.); gavrielf@volcani.agri.gov.il (G.F.); nircarmi@volcani.agri.gov.il (N.C.); \\ irisy@volcali.agri.gov.il (I.Y.); Michal.sharon78@gmail.com (M.S.C.) \\ 2. Department of Agroecology and Plant Health, The Robert H. Smith Faculty of Agriculture, Food and \\ Environment, The Hebrew University of Jerusalem, Rehovot 7610001, Israel \\ 3 Extension Service, Ministry of Agriculture, Israel; shoshw@shaham.moag.gov.il (S.W.) \\ 4 Department of Fruit Tree Sciences, Institute of Plant Sciences, The Volcani Center, P.O. Box 15159, Rishon \\ LeZion 7505101, Israel; \\ 5 Agricultural Research and Development, Central Mountain Region, P.O. Box 104, Tekoa 90908, Israel \\ * Correspondence: noamr@volcani.agri.gov.ili irisy@volcali.agri.gov.il; Tel.: +972-3-9683387
}

\begin{abstract}
The development of new cultivars is important for the profitability of the floriculture industry. There are a limited number of cultivars of Zantedeschia aethiopica, an iconic ornamental cut flower, garden plant and potted plant, due to incompatibility of interspecific crossings within the genus. Most present-day varieties are the result of spontaneous mutations or classical breeding within the species, followed by a long selection process. Breeders are very interested in the development of a time- and cost-effective method for producing new Z. aethiopica cultivars with novel characteristics. Here, Z. aethiopica mutants were generated by treating seeds with 100 Gy of X-ray radiation. The resulting putative mutants were selected based on particular flowering parameters and compared to non-irradiated, control plants. Over two growing seasons, characteristics such as early flowering, flower size and shape, yield and response to soft-rot disease were monitored and considerable variation was observed among the mutated lines. Out of 319 mutants, 20 lines were selected based on their phenotypes and then propagated and further analyzed. Within this group, only two phenotypes displayed at least five improved flowering properties under natural, Mediterranean conditions. The rest displayed two to four desired combinations of flowering traits, some with great commercial potential.
\end{abstract}

Keywords: Zantedeschia aethiopica; X-ray mutagenesis; cultivation; Pectobacterium; mutation breeding

\section{Introduction}

Zantedeschia aethiopica (L.) Spreng. also known as arum lily, or calla lily, is an iconic ornamental herbaceous perennial with high yields that grows well in a variety of soils [1]. The genus Zantedeschia belongs to the family Aracea and is native to southern Africa. Zantedeschia species are cultivated commercially worldwide for cut flowers, garden plants and potted plants [2, 3]. Letty [4] grouped the species into two informal sections: Zantedeschia, containing two species of the evergreen Z. aethiopica and the summer dormant Z. odorata, both with a white spathe and a rhizomatous storage organ; and Aestivae, containing six winter-dormant species with a colored spathe and a tuberous storage organ [4,5]. One of the major threats to Z. aethiopica crops around the world is the soft-rot disease caused by Pectobacterium spp., although Z. aethiopica is more resilient than the colorful Zantedeschia hybrids (Aestivae) group [6-8]. A major factor enabling commercial development of high- 
value cultivars is the species' breeding potential, but unlike members of the Aestivae group, which can be easily crossed through conventional interspecific hybridization, members of the Zantedeschia group may only be crossed with other members of their own species [9]. Moreover, the two groups cannot inter-cross due to plastome-genome incompatibility [9-11].

The market demand for the classical arum flowers with their bright white funnel-like spathe surrounding a yellow, upright spadix is relatively steady, mainly because of their traditional role as wedding and funeral flowers in many cultures. These flowers are exported to the European market from several Mediterranean countries, including Israel. Israeli growers use a single variety, namely Z. aethiopica var. Israeli $(Z A I)$, which naturally blooms during the late winter (weeks 6-18). This variety has never been registered as a commercial cultivar and is derived from several genetic resources of South African origin that have been vegetatively propagated by flower growers over the last decades [12]. The ZAI variety has solid dark green ovate to cordiform leaves, its spathe is white and its spadix is yellow to orange and odorless. Growing a crop that lacks distinctive characteristics and is not uniform is a significant limitation for calla-lily growers in Israel. We hypothesized that by increasing the genetic diversity via mutagenesis, it would be possible to select a unique phenotype for this agricultural niche. Z. aethiopica has been cultivated as a cut flower and propagation material by local producers for more than 50 years, mainly for export to EU markets, taking advantage of the subtropical Mediterranean climate that allows production during the winter season. Although there is a stable demand for this flower, the prices of the cut stems vary significantly, depending on their current availability on the market. Prices start around $0.2 €$ and reach $2.0 €$ between high and low season (Figure 1). Based on the growers' requirements, we defined a few desired traits for the development of a unique local cultivar. The most important of these traits are early flowering, high yield and better tolerance to soft-rot bacteria.

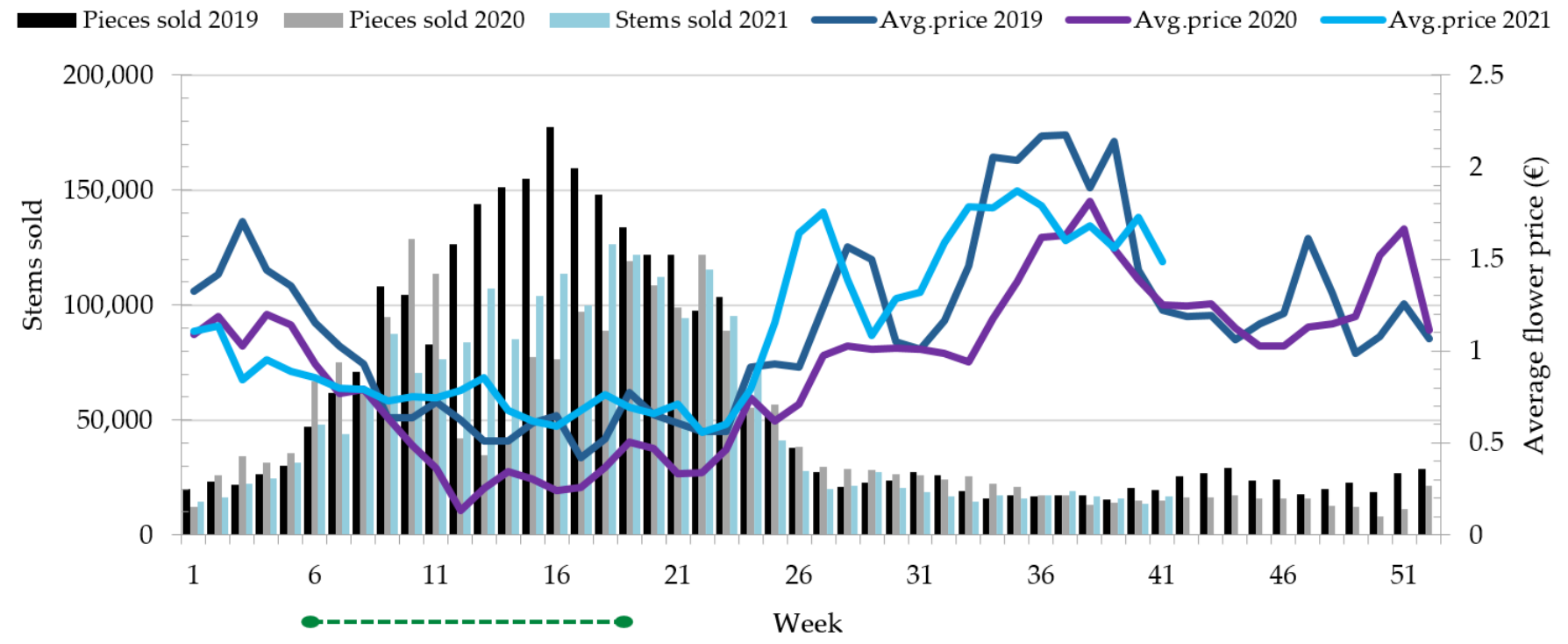

Figure 1. Zantedeschia aethiopica sales in Royal FloraHolland over time (weeks) during the 2019, 2020 and 2021 marketing seasons. The average marketing price is defined on the right-hand y-axis $(€)$ and presented by the solid lines. The numbers of stems sold are presented as bars and defined on the left-hand $y$-axis. The green dashed line under the $x$-axis represents the flowering season of the $Z A I$ variety when grown under outdoor conditions in Israel. The data are provided with permission from Royal FloraHolland. 
Classical breeding requires a great deal of time and labor and the presence of a typical phenotype in the parental accession lines is a prerequisite for success. Mutation induction can introduce new phenotypes, which are not present in the parental line, and accelerate the selection procedure. This approach holds good potential for increasing genetic variation in Z. aethiopica, overcoming the inability to cross it with other Zantedeschia species. Mutation breeding has been proven successful throughout the development of hundreds of cultivars in the ornamental-plant industry, especially among vegetatively propagated species. Ionizing radiation involving $X$-rays, gamma rays or neutrons and chemical mutagens have all been used to improve ornamental crops [13]. The key factor in irradiation of plant material is the amount of radiation energy (dose) absorbed by the tissue. The dose is measured in units of Gray (Gy), with one Gy equal to the absorption of 1 joule of energy per kilogram of irradiated material. Doses of radiation above $10 \mathrm{kGy}$ are considered to be high, medium doses are between 1-10 kGy and lower doses are less than $1 \mathrm{kGy}$. Irradiation of seed material usually requires doses between 60-700 Gy [13]. Successful mutagenesis is also dependent on the plant material being used (e.g., stem cuttings, cell suspensions, callus cultures or seeds) and varies between different plant species. Thus, each system needs to be calibrated, in order to determine the optimal irradiation dose before application [13-15]. The resulting mutant plants with altered phenotypes make the selection process straightforward and easy. Hence, mutation techniques have become an important tool for breeding ornamental plants with hundreds of varieties including Chrysanthemum, Alstromeria, Dahlia, Rosa, Begonia, Dianthus, Azalea and others [13].

The main objective of the present study was to select an early flowering Z. aethiopica cultivar with high yield and improved tolerance to soft rot. The traditional use of vegetative propagation in this ornamental crop has made its genetic variability relatively low. To improve the genetic variation of Z. aethiopica and select for desired phenotypes, seeds of Z. aethiopica of South African origin (Seeds and All, Port Elizabeth, South Africa) were irradiated using X-ray technology. This was followed by a phenotype-selection procedure and the identification of early-flowering, high-yielding and soft rot-tolerant cultivars under natural Mediterranean conditions.

\section{Materials and Methods}

\subsection{Plant Materials, Bacterial Strains and Growth Media}

Three seed varieties of Z. aethiopica were imported: a local variety from Seeds and All', Port Elizabeth, South Africa; 'Pink Mist' from Seeds for Africa, Cape Town, South Africa; and unregistered variety, 'Thompson and Morgan', from Ipswich, United Kingdom. In addition, a local variety grown in Israel (ZAI) was acquired from a local grower. Pectobacterium brasiliense strain Pb1692 (originally isolated from potato) was used for infection assays. Pb1692 was cultivated in lysogenic broth (LB; Difco Laboratories, Detroit, MI, USA) at $28^{\circ} \mathrm{C}$ and inoculated in minimal medium (MM) as described [16]. Murashige and Skoog (MS), agar medium and vitamins were purchased from Duchefa (Duchefa Biochemie, The Netherlands).

\subsection{Germination rates of $Z$. aethiopica varieties}

Z. aethiopica seeds of all varieties (30-175 seeds, depending on the seed source) were soaked in distilled water overnight, sown in $30-\mathrm{mL}$ Styrofoam containers filled with RAM8 planting mix (Section 2.4), and placed in a greenhouse that was kept at $25^{\circ} \mathrm{C}$ with natural daylight. In addition, disinfected seeds were sown on MS agar plates as described in [17]. Seedlings grown from seeds that exhibited early germination were counted and their survival rates were calculated.

\subsection{Mutagenesis}


Z. aethiopica seeds were irradiated in the Life Sciences Core Facilities of the Weizmann Institute of Science (Rehovot, Israel), using the X-RAD 320 (North Branford, CT, USA) system that provides a highly homogenous beam and a precise dose of radiation.

\subsection{Growth Conditions and Field Experiment}

During September 2016, 3,000 Z. aethiopica seeds (radiated and control) were soaked in water overnight and then sown in 30-mL Styrofoam containers filled with RAM8 planting mix (Tuff Marom Golan, Golan Heights, Israel), which consisted of $20 \%$ peat moss, $70 \%$ coconut fiber and $10 \%$ compost (by volume). The containers were placed in a greenhouse with natural daylight, which was kept at $25^{\circ} \mathrm{C}$. After germination (in December 2016), 319 radiated and 72 untreated control seedlings grown from early-germinating seeds were planted in 500-mL plastic pots filled with the same planting mix for further growth. The pots were placed in a net-house with $10 \%$ shade, where they were kept under natural light/temperature conditions. The plants were irrigated and fertilized according to local commercial recommendations. During July 2017, the plants were transplanted into 10-L plastic pots filled with the same planting mix. Each plant represented an individual line originating from a single seed, labeled Ca for "control" or CaX for "irradiated" and each plant was also assigned a serial number. From August 2017 through June 2018 (first growing season), growth and flowering parameters were monitored for each of these plants, after which, selected lines were isolated. During August 2018, rhizomes from each selected line were split into 10 comparable units, which were planted and grown under the same conditions. From August 2018 through June 2019 (the second growing season), growth and flowering parameters were again monitored for each line.

\subsection{Collection of Data About Plant Traits}

All flowering data (i.e., early flowering, flower yield, flower size and shape) were collected separately for each individual plant and for each line over two consecutive growing seasons. The qualitative parameter of flower scent was treated as a binary parameter (i.e., present or not present). Data calculations and graphs were generated using Excel16 (Microsoft, Redmond, WA, USA). Venn diagrams were generated using VennDIS Ver.1.2 [18].

\subsection{Tolerance to Pectobacterium}

The infection assays were conducted as described previously [19], with minor modifications. All assays were performed during the second growing season, starting from rhizome division. The second fully open young leaves were harvested, soaked in $0.7 \%$ sodium hypochlorite $(20 \mathrm{~min}$ ) for external disinfection, and then washed twice in sterile distilled water. Leaf discs (20 mm in diam.) were then excised, pierced at the center with a sterile $10-\mu \mathrm{L}$ tip and placed onto Petri dishes containing $20 \mathrm{~mL}$ of half-concentrated MS supplemented with $6 \mathrm{~g}$ of agar per mL. Inoculation was performed using $10-\mu \mathrm{L}$ of midlog-phase cultures of Pb1692 at $10^{9}$ colony-forming units (CFU) $\mathrm{mL}^{-1}$ (OD600 0.1). Prior to inoculation, the culture was cultivated in $\mathrm{LB}$ at $28^{\circ} \mathrm{C}$, washed twice with minimal media (MM) and then re-suspended in MM. For the untreated control, the leaf discs were inoculated with $\mathrm{MM}$ alone. The plates were incubated at $28^{\circ} \mathrm{C}$ for $21 \mathrm{~h}$. After that incubation, they were scanned and the necrotic area was measured using ImageJ software [20] and divided by the total area of the disc to calculate the percentage of decayed tissue. Leaf discs in which no infection was observed were omitted from the final calculations for lines in which high levels of infection $(>75 \%)$ were observed among the tested leaf discs. The assays were conducted on 30-40 leaf discs from three different plants for each line. All virulence assays were repeated twice. 


\subsection{Statistical Analysis}

Multiple comparison tests using the Dunnett's method were conducted as subsequent tests. JMP-Pro version 13.0 (SAS Institute Inc., Cary, NC, USA) statistical software was used for statistical processing and the significance probability was set at $p \leq 0.001$.

\section{Results}

\subsection{Choice of Propagation Material}

We imported Z. aethiopica seeds from three sources (two sources in South Africa and one in England). The germination rates of the imported seeds and seeds of the local variety grown in Israel ( $Z A I)$ were examined. The seeds from the company Seeds and All (South Africa) germinated with the highest efficiency (88\%) in both the planting mix and the Petri dishes, and exhibited highly uniform germination. According to the manufacturer, these seeds are produced from recognized accessions and maintain high uniformity over the years. Therefore, we decided to continue with this seed source. The ZAI seeds exhibited the least uniform germination.

\subsection{Calibration of the Dose of X-Ray Irradiation}

Calibration of the dose of $X$-ray irradiation was performed on the chosen seeds. To determine the optimal dose of radiation that would allow a survival rate of $20-50 \%$ (LD50) of the treated seeds, 50 seeds in five Petri dishes (250 seeds per dose) were irradiated in an X-RAD 320 system. As expected, the results revealed a negative correlation between the radiation dose and the seed survival rate $\left(R^{2}=0.967\right)$. A dose of $50 \mathrm{~Gy}$ with an exposure time of $10 \mathrm{~min}$ was the best fit for an LD50 (46\%) survival rate. To increase phenotypic variation, we chose 20 min of exposure (100 Gy) as the standard dose for the experiment. The radiation dose and the duration of exposure along with the survival rates of the treated seeds are presented in Table 1 and Figure S1.

Table 1. Calibration of the radiation dose for Zantedeschia aethiopica seeds, duration of exposure and survival rates of treated seeds. The chosen conditions are marked in bold; Gy = Gray.

\begin{tabular}{ccccccccc}
\hline Duration (Min) & $\mathbf{0}$ & $\mathbf{2}$ & $\mathbf{4}$ & $\mathbf{1 0}$ & $\mathbf{2 0}$ & $\mathbf{1 0 0}$ & $\mathbf{1 5 0}$ & $\mathbf{2 0 0}$ \\
\hline Radiation dose (Gy) & 0 & 10 & 20 & 50 & $\mathbf{1 0 0}$ & 500 & 750 & 1000 \\
Survival rate (\%) & 70 & 75 & 59 & 46 & $\mathbf{1 6}$ & 0 & 0 & 0 \\
\hline
\end{tabular}

\subsection{X-ray Radiation of Z. aethiopica Seeds}

X-ray mutagenesis with $100 \mathrm{~Gy}$ of radiation was performed on 1800 new, dry Z. aethiopica seeds imported from South Africa ( $\mathrm{CaX})$. Then, the seeds were sown in planting mix. One hundred and eighty seeds from the same origin $(\mathrm{Ca})$ were used as a control and were sown under the same conditions without treatment. The survival rate of the irradiated seeds was $17.72 \%$ (319 plants) and the survival rate of the control seeds was $40 \%$ (72 plants). All surviving seeds from both groups were transferred to 10-L pots filled with planting medium for continued monitoring of growth and development.

\subsection{First-Season (2017-2018) Mutant Phenotypes}

The season in Israel for outdoor growing of Z. aethiopica (ZAI) is autumn to spring (September to May), while the flowering season is from February through the beginning of May (a 12-week period). During the first growing stage (before flowering), a few individual mutant lines $(\mathrm{CaX})$ showed morphological changes that differentiated them from the non-radiated control lines $(C a)$. The differences were mainly in leaf shape, number of stems and mosaic stains, as presented in Figure 2. However, most differences were detected during the flowering season. These included differences in flowering time, number of flowers per plant (yield), flower shape and color, flower size and floral scent (Figure 
S2). Some of the mutants exhibited combinations of desirable traits (Figure 3a). A summary of the phenotypes is provided, based on pair-wise intersections (Table 2, Figure 3). Out of 319 CaX lines, about 6\% (20 lines) exhibited valuable traits and were thus chosen for further analysis. Most of those selected lines presented several desired phenotypes, some with a combination of five or six desirable traits (CaX100 and CaX215, respectively; Figure 3).

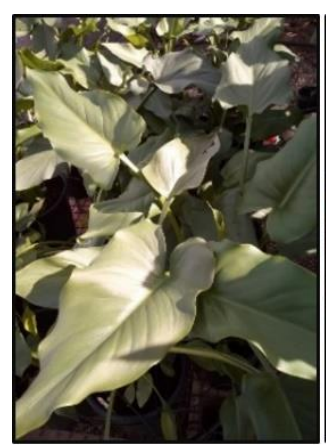

(a)

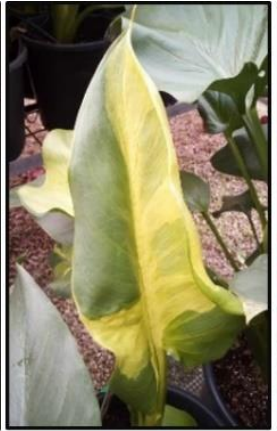

(b)

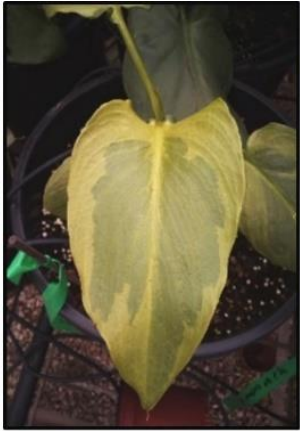

(c)

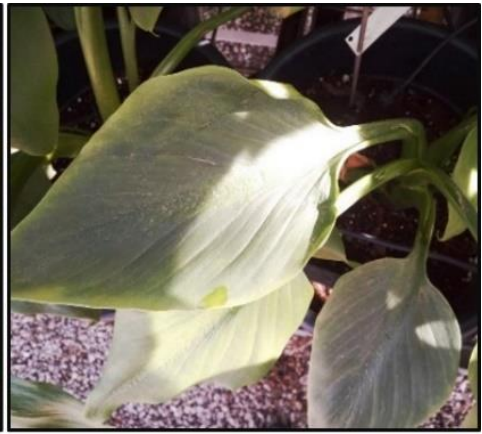

(d)

Figure 2. Morphological changes in the mutated lines during the first growing season. (a) Leaves from Z. aethiopica control line $(\mathrm{Ca})$ that were not irradiated. (b-d) Z. aethiopica mutant lines $(\mathrm{CaX})$ that were irradiated with $X$-rays; each image represents an individual line. Changes included leaves with (b, c) mosaic-like appearance, (b) distortions and (d) rounded leaves. These morphological changes indicate that mutations have occurred at different positions.Table 2. Pairwise intersection of nine traits observed in 143 mutant lines (CaX) during the first season (2017-2018). The intersection is displayed as a minimal triangular matrix, in which the different numbers represent mutant lines that share a specific trait. The total number of mutant lines that exhibited the trait is written in parentheses next to each trait. High yield means high number of flowers produced, fl. for flowering and F. for flowers. "Scent" is related to presence of scent in flowers and "Mosaic" to a mosaic appearance on the leaves.

\begin{tabular}{ccccccccc}
\hline & $\begin{array}{c}\text { Early } \\
\text { fl. }\end{array}$ & $\begin{array}{c}\text { Large } \\
\text { F. }\end{array}$ & $\begin{array}{c}\text { Small } \\
\text { F. }\end{array}$ & $\begin{array}{c}\text { Desired F. Flower } \\
\text { shape }\end{array}$ & $\begin{array}{c}\text { Scent } \\
\text { (53) }\end{array}$ & $\begin{array}{c}\text { spathe } \\
(\mathbf{2 3})\end{array}$ & $\begin{array}{c}\text { Dark } \\
\text { spadix } \\
(\mathbf{2 9 )}\end{array}$ & $\begin{array}{c}\text { Mosaic } \\
\text { (3) }\end{array}$ \\
\hline High yield (10) & 4 & 4 & 2 & 8 & 6 & 2 & 3 & 0 \\
Early fl. (34) & & 12 & 8 & 19 & 14 & 7 & 1 & 0 \\
Large F. (43) & & & 1 & 26 & 22 & 7 & 6 & 0 \\
Small F. (39) & & & & 17 & 8 & 7 & 8 & 0 \\
Desired F. shape & & & & 32 & 11 & 18 & 1 \\
$\quad \begin{array}{c}\text { (82) } \\
\text { Scent (53) }\end{array}$ & & & & & 5 & 14 & 0 \\
Shades on spathe & & & & & & 1 & 0 \\
$\quad$ (23) & & & & & & & 0 \\
Dark spadix (29) & & & & & & & & \\
\hline
\end{tabular}




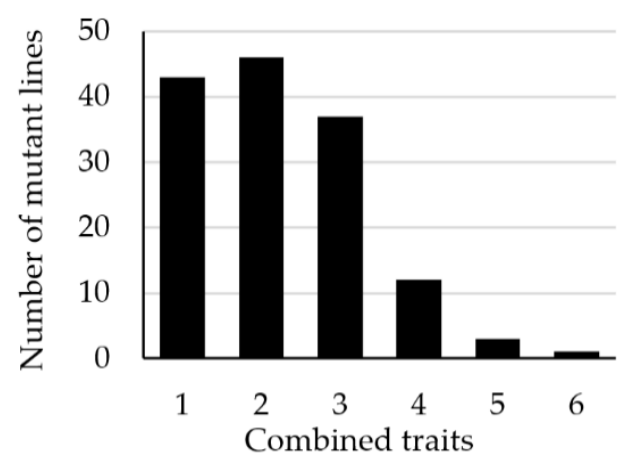

(a)

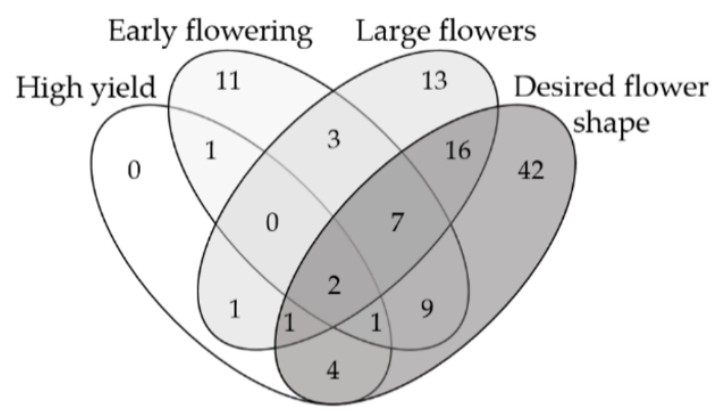

(b)

Figure 3. Summary of mutated Z. aethiopica $(\mathrm{CaX})$ phenotypes from the first season. (a) The number of common traits observed among 142 different mutant lines (CaX). (b) Venn diagram calculated based on the four most desired properties of the mutated lines from the first season.

\subsection{Phenotypes of Mutated Plants During the Second Growing Season (2018-2019)}

To ensure that the traits of each of the mutated selected lines $(\mathrm{CaX})$ were stable, bulbs from each of the lines were split into 10 similar units and each unit was grown separately for the next season (i.e., the second season), as described previously. The control consisted of two different non-irradiated Z. aethiopica lines $(C a)$, as well as two different $Z A I$ lines, whose bulbs were split similarly to those of the $C a X$ lines. The two $C a$ control lines exhibited a stable phenotype in the first growing season, consistent with the phenotype described by the supplier (Seeds and All). During the second growing season (2018-2019), the phenotypic combinations of the selected mutant lines $(\mathrm{CaX})$ were compared to those of the control lines ( $\mathrm{Ca}$ and $\mathrm{ZAI}$ ).

During the second growing season, all of the chosen lines (10 clones each) were inspected for the following traits: flowering time, number of flowers per mutant line (yield), flower size, flower shape, presence of floral scent, color of flowers, leaf patterns and tolerance to Pectobacterium infection. All of those traits were compared to those observed in the control lines. Examples of the variability in the flowers of the selected mutant lines from the second season are presented in Figure 4 and Figure S2.

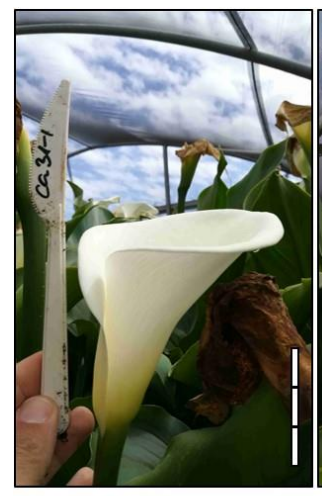

(a)

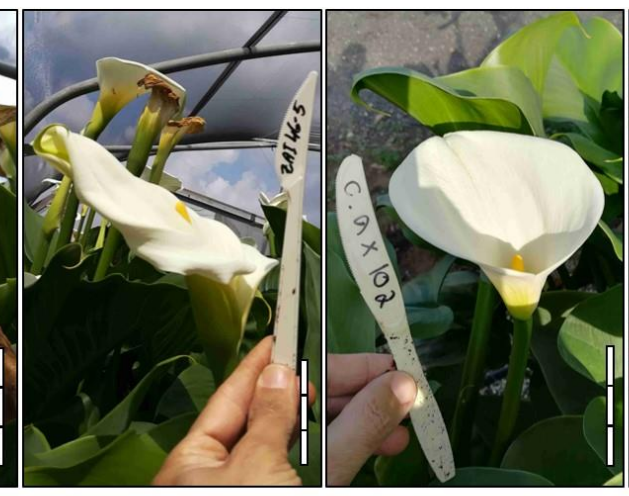

(b) (c)

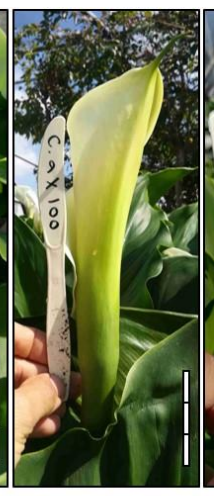

(d)

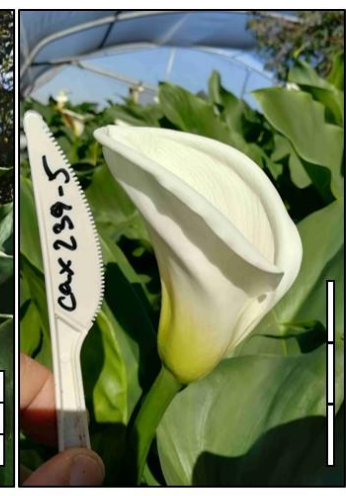

(e)

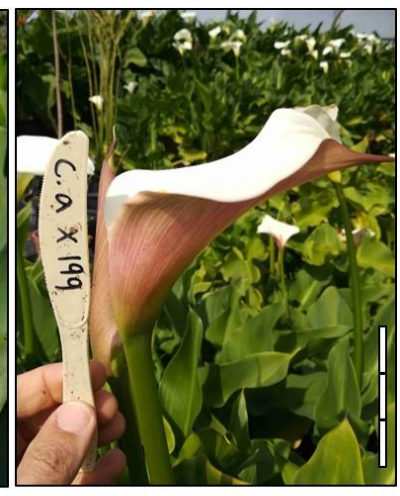

(f)

Figure 4. Flower diversity of mutated lines during the second growing season (2018). (a) Control line Ca. (b) The Israeli ecotype ZAI. (c-f) Flower variations observed in the X-ray irradiated mutant lines, $\mathrm{CaX}$. Numerous flower formations were observed among the mutants. Some of them had (c) small and rounded shape flowers, (d) very large flowers (e) with curved borders, and/or (f) a colored spathe. The vertical white dashed bar on the right of each image represents a length of 6 $\mathrm{cm}$. 
In the second season, $80 \%$ of the phenotypic characteristics that were observed in the first year appeared once again in the same lines. Ten percent retained some of the characteristics observed in the first year and $10 \%$ were completely different in the two years.

\subsubsection{Early and Late Flowering}

To monitor early flowering phenotypes, we established two definitions: onset of flowering (OOF) - the flowering time of the first $10 \%$ of the total flowers for each mutant line and early flowering (EF) - mutant lines in which OOF was 6 weeks earlier than that of the control lines. We used a 6-week period to overcome the natural variation in OOF. As illustrated in Figure 5, the OOF of the control lines took place during March 2019, while the timing of the OOF of the mutant lines ranged from November 2018 through March 2019. Therefore, in the second season, the EF was calculated based on OOF obtained earlier than January 2019. Among the mutant lines, 50\% had an OOF from February 2018, and $25 \%$ were EF; one of the mutants' OOF was as early as 3 months before that of the control line (Figure 5).

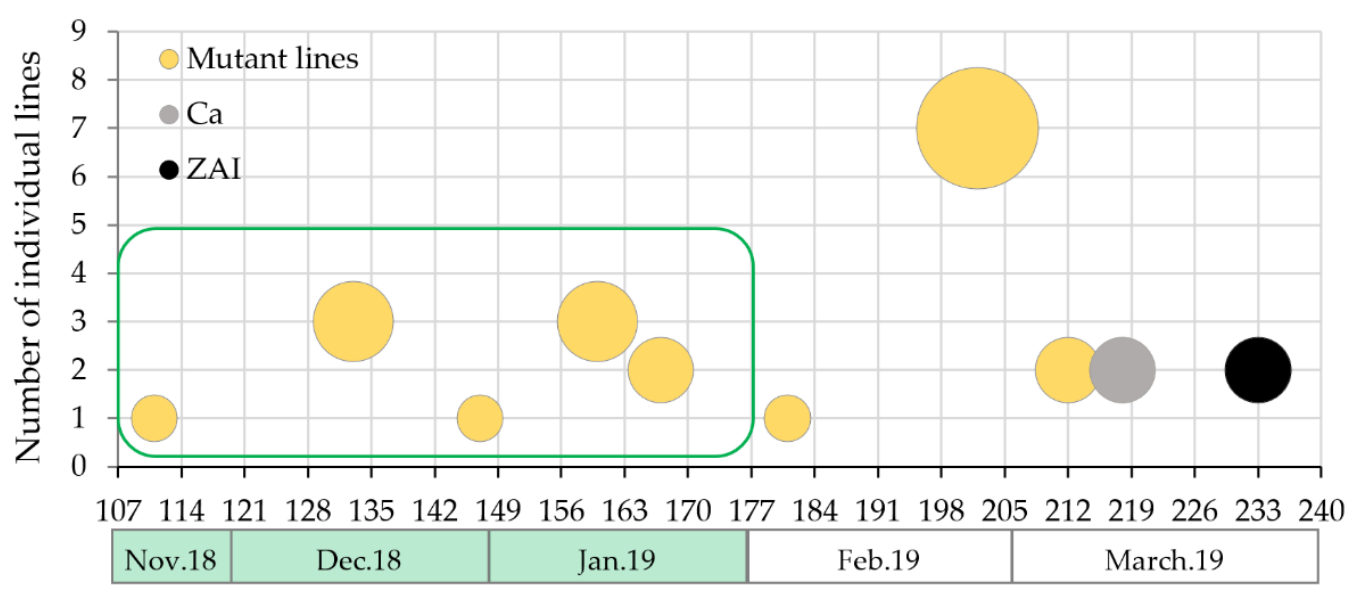

Appearance of first 10\% of flowers [Days from planting]

Figure 5. Onset of flowering (OOF) of 20 mutated Z. aethiopica lines compared to the control line during the 2018-2019 growing season. Each bubble represents a different group of mutant lines in which more than $10 \%$ of the total flowers from that line appeared and the bubble size represents the number of lines ( $\mathrm{y}$-axis) that flowered at that time (x-axis). Mutant lines ( $\mathrm{CaX})$ are presented in yellow, the control (Ca) is shown in gray, and the local Israeli line ZAI is shown in black. Time is measured as days from planting and the months are listed below the x-axis, with the green background and the green box representing the period defined as early flowering.

\subsubsection{Flower Yield}

Flower yield was measured as the average amount of flowers produced by all of the plants of each line during the second growing season (2018-2019). As shown in Figure $6 a$, the average yield of the control lines (Ca) was less than two flowers per plant, similar to $25 \%$ of the mutated lines. Only $15 \%$ of the mutated lines had lower yields (about one flower per plant). Yet, $60 \%$ of the selected mutated lines had higher yields (i.e., two to four flowers per plant) and four of the mutated lines had significantly higher yields $(\mathrm{p}<0.001)$ of more than three flowers per plant (Figure 6a). The local Israeli line (ZAI) had significantly lower yields than the South African lines (Ca, CaX).

\subsubsection{Flower Size}

The flower-size trait was based on the full length of the spathe before opening and was divided into four groups: (1) small - flower length of $5 \mathrm{~cm}$ or less; (2) medium - flower length of 6-10 cm (this included the Ca and ZAI groups); (3) large - flower length of 11-14 
$\mathrm{cm}$ and (4) extra-large size - flowers longer than $14 \mathrm{~cm}$. As shown in Figure 6b, one mutated line had significantly smaller flowers than the control, 11 lines had significantly larger flowers than the control and four lines had extra-large flowers that were more than $14 \mathrm{~cm}$ long.

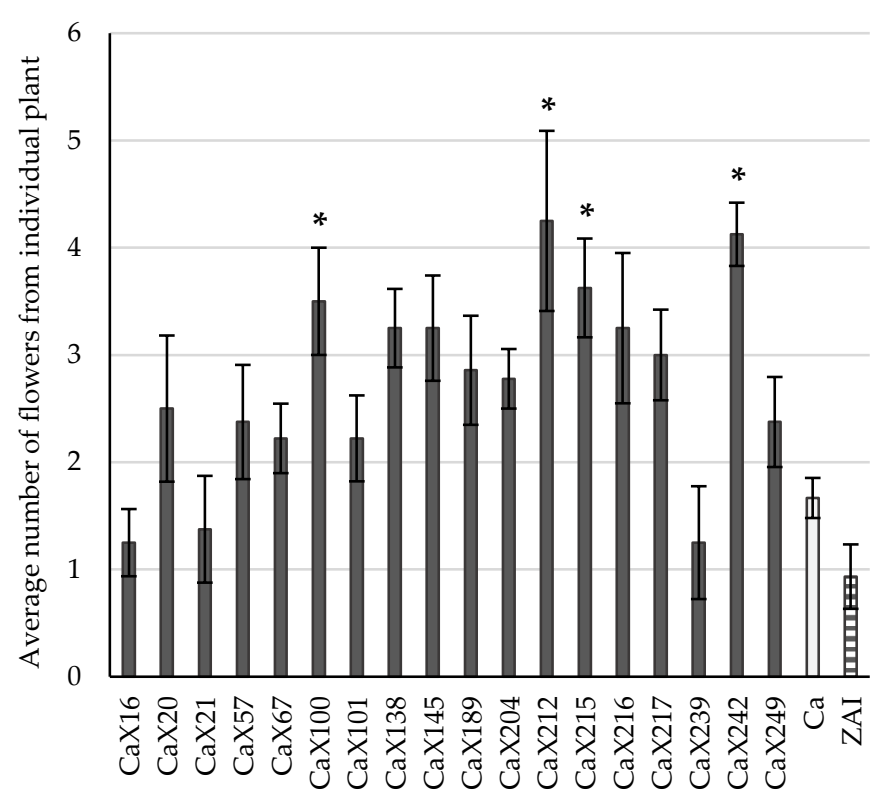

(a)

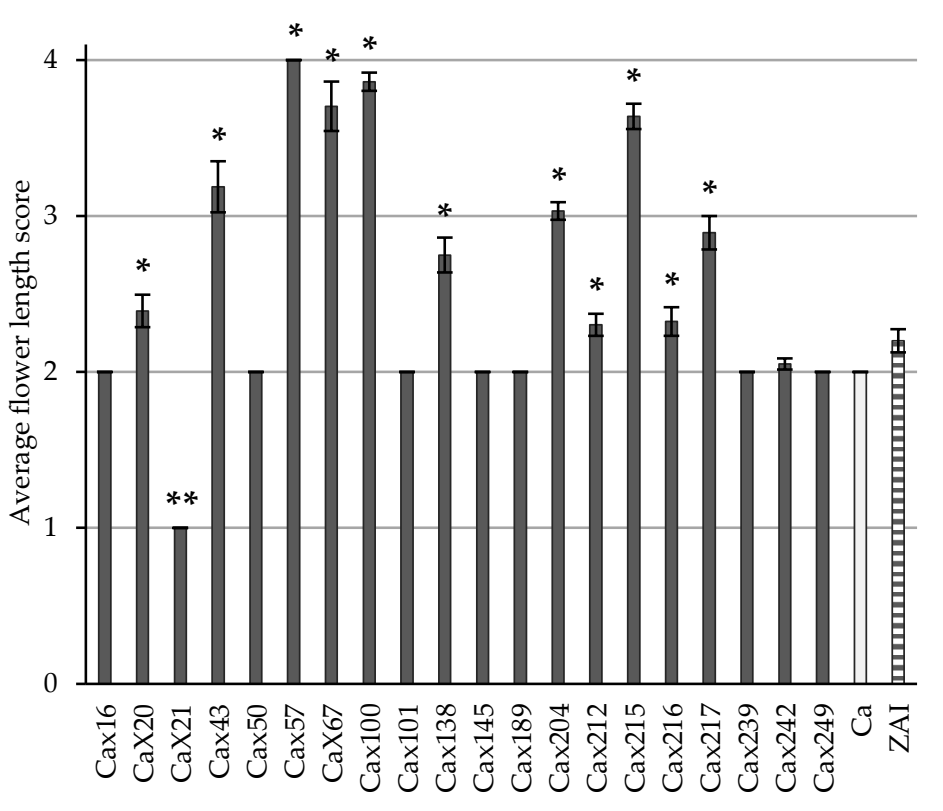

(b)

Figure 6. (a) Flower yield and (b) flower size obtained from the selected Z. aethiopica mutated lines compared to the control lines during the second growing season (2018-2019). (a) The average numbers of flowers per plant from each line are presented as bars with standard errors indicated for each column. (b) The average flower sizes of the selected lines are represented in length groups with the following score: small $(<5 \mathrm{~cm})$ group 1, medium $(6-10 \mathrm{~cm})$ group 2, large $(11-14 \mathrm{~cm})$ group 3, and extra-large $(>14 \mathrm{~cm})$ group 4 . The mutated lines are labeled " $\mathrm{CaX}$ " and control lines are labeled " $\mathrm{Ca}$ ". The Israeli local line is labeled " $\mathrm{ZAI}$ ". Values significantly greater than the corresponding control values are marked with a single asterisk $\left(^{*}\right)(p<0.001)$. Double asterisks $\left(^{* *}\right)$ indicate significantly smaller size $(p<0.001)$.

\subsubsection{Desired Flower Shape}

The mutated lines had a variety of flower shapes. To define the preferred flower shape, we focused on the side and upper projections. With this method, we defined four projections, two side views and two top views. The side view included the open or closed spathe and the top view included the drop or rounded shape of the spathe, as presented in Figure 7. The desired flower shape consists of a combination of closed and rounded shape (Figure $7 \mathrm{~b}, \mathrm{~d}$ ) with the spathe appearing as a smooth cup. Seventy-five percent of all of the selected mutated lines had cup-shaped flowers.

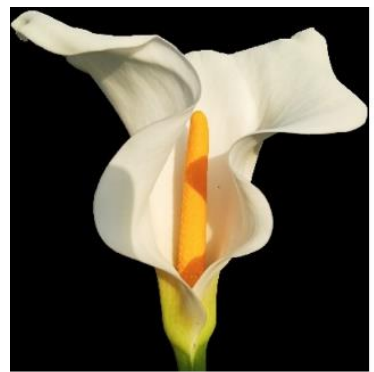

(a)

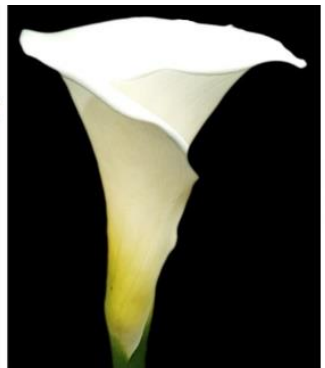

(b)

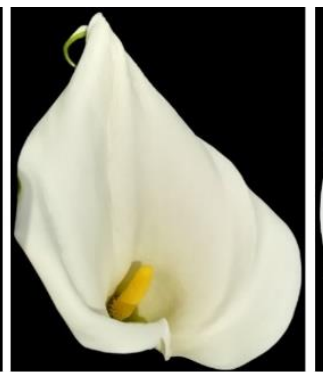

(c)

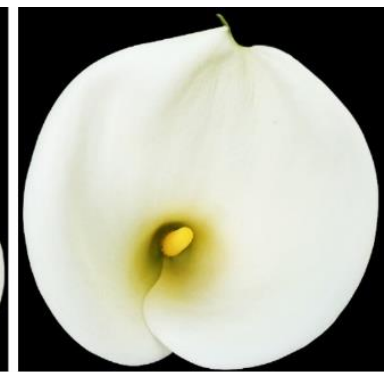

(d) 
Figure 7. Examples of flower shapes. Side view: (a) open or (b) closed. Top view: (c) drop shape or (d) rounded shape. The most desired flower shape is a combination of closed and rounded shapes (b and d).

\subsubsection{Tolerance to Pectobacterium Infection}

Another desired property for Zantedeschia plants is tolerance to soft-rot disease caused by bacterial pathogens of the genus Pectobacterium. Since it would have been both time- and labor-intensive to scan large numbers of individual mutated lines during the first growing season, we decided to focus on the 20 best mutated lines (CaX) from the second growing season. As Cax plants were generated from irradiated seeds, it was important to assess the natural variation of disease tolerance among the control lines $(\mathrm{Ca})$. To do so, we analyzed 19 individual $C a$ lines by inoculating leaf discs of each line with $10^{8}$ CFU of Pectobacterium strain Pb1692. The relative size of the decayed area was recorded at $21 \mathrm{~h}$ post-inoculation (Figure 8a). The decay area (\% of total area) was calculated using 19 individual $\mathrm{Ca}$ lines and compared to that observed on the local Israeli line ZAI (Figure $8 \mathrm{~b})$. The average extent of decay of the $C a$ lines was significantly lower $(18 \%)$ than that of the local Israeli line ZAI (28\%).

Next, the infection assays were performed for all of the selected CaX lines, two Ca lines and the local Israeli line ZAI were used as controls (Figure 8d). Compared to the $\mathrm{Ca}$ lines, the mutated lines exhibited greater variability; eight Cax lines were significantly more tolerant to $\mathrm{Pb} 1692$ infection and two lines that were significantly more susceptible, similar to ZAI (Figure 8d). A comparison of the degree of variation in each of the two groups revealed that the mean and median values for the two groups were very similar, but that there was greater variability among the irradiated lines (Figure 8e).

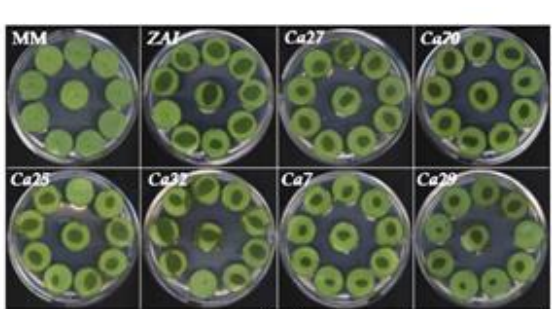

(a)

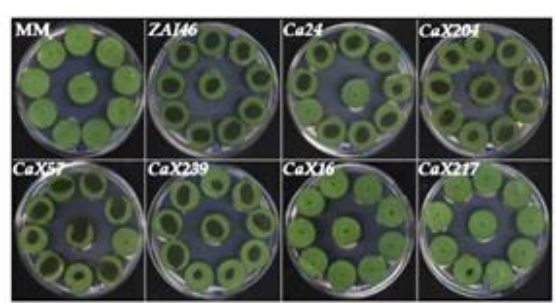

(c)

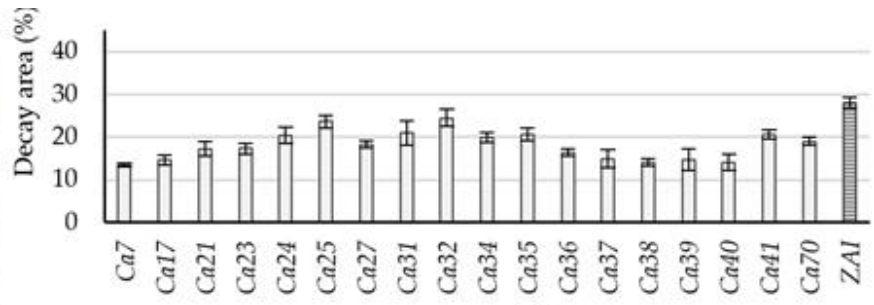

(b)
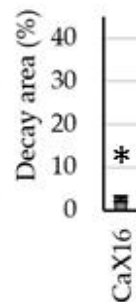

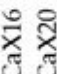

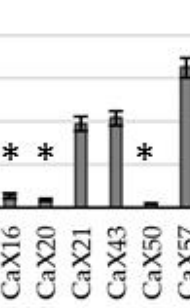

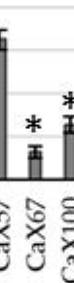

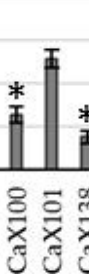
lii

Figure 8. Assessment of Pectobacterium brasiliense Pb1692 infection of leaf discs of the control (Ca) and selected mutated lines (Cax) of Zantedeschia aethiopica. Leaf discs were pierced in the center and inoculated with $10 \mu \mathrm{L}$ of $\mathrm{Pb} 1692\left(10^{9} \mathrm{CFU} \mathrm{mL} \mathrm{m}^{-1}\right)$. Infection was measured as area of decayed tissue (\%), $21 \mathrm{~h}$ after inoculation and incubation at $28^{\circ} \mathrm{C}$. (a) A representative photo of the infected leaf discs of the Ca lines. (b) Percentage of tissue area covered by decay among the Ca lines. (c) A representative photo of the infected leaf discs of the mutated lines (CaX). (d) Percentage of tissue area covered by decay among $20 \mathrm{CaX}$ lines, compared to the average of the control lines (Ca24 and Ca31) and ZAI. In (a) and (c) the line labels are marked on the upper left corner of each plate. MM stands for non-inoculated control (minimal medium). The local Israeli line is marked as ZAI. The bars represent average values with standard errors. Lines that are significantly different from the control are marked with asterisks $(p<0.001)$. (e) Comparison of variation between the $\mathrm{Ca}$ lines and the $\mathrm{CaX}$ lines, in terms of the percentage of tissue area covered by decay,; " $\mathrm{x}$ " is the average and "_" is the median.

\subsection{Summary of the Mutated Phenotypes (2018-2019)}


At the end of the second growing season, we could detect mutant lines with different desired traits, most of which $(75 \%)$ had more than two of the desired traits. Two mutated lines (10\%) had five or six of the desired traits (CaX100 and CaX215, respectively), 30\% had four of the desired traits, $10 \%$ had three of the desired traits and $30 \%$ had two of the desired traits. A single line (CaX101) did not exhibit any of the desired traits. A summary of all of the examined traits in the 20 selected mutated lines is presented in Table 3.

Table 3. Summary of traits observed during the 2018-2019 season among the selected X-ray-mutated lines. $(\mathrm{CaX})$. + indicates presence of the trait.

\begin{tabular}{|c|c|c|c|c|c|c|c|}
\hline $\begin{array}{l}\text { Mutant } \\
\text { line }\end{array}$ & High yield & $\begin{array}{l}\text { Early flow- } \\
\text { ering }\end{array}$ & $\begin{array}{c}\text { Pectobacterium } \\
\text { tolerance }\end{array}$ & $\begin{array}{l}\text { Large } \\
\text { flowers }\end{array}$ & $\begin{array}{c}\text { Desired } \\
\text { flower } \\
\text { shape }\end{array}$ & $\begin{array}{l}\text { Scent in } \\
\text { flowers }\end{array}$ & $\begin{array}{l}\text { Total inter- } \\
\text { section oc- } \\
\text { currences }\end{array}$ \\
\hline CaX100 & + & + & + & + & + & + & 6 \\
\hline CaX215 & + & + & & + & + & + & 5 \\
\hline CaX189 & & + & + & & + & + & 4 \\
\hline CaX242 & + & + & & & + & + & 4 \\
\hline CaX138 & & & + & + & + & + & 4 \\
\hline CaX20 & & & + & + & + & + & 4 \\
\hline CaX67 & & & + & + & + & + & 4 \\
\hline CaX217 & & + & + & + & + & & 4 \\
\hline CaX21 & & + & & & + & + & 3 \\
\hline CaX57 & & & & + & + & + & 3 \\
\hline CaX212 & + & + & & & & & 2 \\
\hline CaX16 & & & + & & + & & 2 \\
\hline CaX50 & & & + & & & + & 2 \\
\hline CaX239 & & & & & + & + & 2 \\
\hline CaX249 & & & & & + & + & 2 \\
\hline CaX43 & & & & + & & + & 2 \\
\hline CaX204 & & & & + & & & 1 \\
\hline CaX216 & & & & & + & & 1 \\
\hline CaX145 & & & & & + & & 1 \\
\hline CaX101 & & & & & & & 0 \\
\hline
\end{tabular}

\section{Discussion}

The main goal of this study was to increase the genetic variation in Z. aethiopica, in order to select a Z. aethiopica variety that is better suited for Mediterenian growers. We hypothesized that the best way to promote this aim would be to select a better-performing cultivar under the local winter conditions, as winter is the preferred growing season for Israeli growers. Although the use of ionized radiation is an established method for inducing mutations in cultivated plants [13], as far as we know, this is the first time that X-rays have been used to induce mutation in Z. aethiopica seeds. As Z. aethiopica is propagated vegetatively, this method of cultivation is especially feasible [13]. X-rays and gamma radiation cause a mixture of gene mutations and chromosomal mutations or rearrangements and the effects of this method are dependent on the dose of radiation [21]. In the present study, the radiation dose was set at $100 \mathrm{~Gy}$, to efficiently select for potentially valuable $Z$. aethiopica seeds. In previous studies, similar doses of radiation were found to be effective for mutagenesis of other crops, including ornamental crops [13].

Here, we have created 319 individual mutant lines (CaX), from which we have selected and further propagated 20 lines that exhibit some of the desired traits that we defined: higher flower yield, early flowering time, large flowers with the desired shape, and tolerance to soft rot caused by Pectobacterium [8, 22-24]. Most of the selected varieties had more than one of these traits (i.e., two to six of these traits), resulting in greater potential for commercialization. The mutated lines we have produced and examined have a variety 
of desirable traits, which were found to be stable even after two consecutive growing seasons. Some of the traits that we examined (e.g., tolerance to soft rot) have also been examined in other Z. aethiopica breeding programs that employed a classical breeding approach [25].

Higher yields can easily improve growers' incomes without increasing other production expenses. Here, four mutated lines had flower yields that were significantly higher than those of the controls. None of the selected mutated lines yielded significantly less than the controls.

Early flowering may contribute to yield, as it extends the flowering season. Moreover, it allows marketing during the period that includes Christmas, Valentine's Day and Easter. The winter season in the northern hemisphere is the time of year when competition from other producers in the market is relatively low and prices per flower stem are relatively high. Early flowering may also minimize the amount that growers spend on chemical treatments, which are required when temperatures rise, as well as the amount spent on growth regulators, which are currently applied at least twice during the growing season. Our study identified five varieties that flowered significantlly earlier than the local variety $(Z A I)$ and the non-irradiated $Z$. aethiopica $(\mathrm{Ca})$. All of these early-flowering phenotypes flowered during the period of peak flower prices (i.e., marketing weeks 46-52, Figure 1).

New flower phenotypes may increase the flower's commercial value by virtue of their uniqueness. Several Z. aethiopica cultivars developed using classical breeding and selection are recognized worldwide. These include the dwarf selection 'Childsiana', a pink spathe selection 'Pink Mist', and the very popular 'Green Goddess', which has a green spathe. 'Colombe de la Paix' was selected for its abundant flowering and the cold-tolerant cultivar 'Crowborough' was selected for its cold tolerance. A more recent example of a classically bred and selected cultivar is 'Deja $\mathrm{Vu}^{\prime}$ ', the first calla lily variety registered in Mexico that has three colors in its spathe: white, pink and green [26].

Another example comes from a Korean breeding program that has focused on resistance to soft rot as the most important breeding trait [25]. That program is based on the evaluation of resistance levels and conventional crossings of resistant accessions. Using this approach, two soft rot-resistant lines were selected, 'Silky White' and 'Mont Blanc', which also exhbit good flowering characteristics, homogeneity and stability [25]. Greater tolerance to Pectobacterium can help growers in the battle against soft rot, especially in warmer climate zones. This trait is highly-ranked among Z. aethiopica growers who are coping with climate change and unexpected heat waves that occur even during the coldest time of the year in the Middle East. The warm conditions that already prevail in Israel present a challenge for soft-rot control [27]. We used Pcb1692, a type strain of P. brasiliense, which is a rising soft rot bacterium under warm conditions, to examine tolerance to soft rot. Soft rot is a major problem for the production of economically important crops around the world [28]. Here, we demonstrate that controlled exposure to X-ray radiation can introduce a range of tolerance levels to Pectobacterium. Forty percent of the selected mutated lines were significantly more tolerant to Pectobacterium than the control.

Another important trait is flower appearance, which we have divided into flower shape, size and differences in color. We have included this quality as part of our prefered selection for new cultivars, by focusing on large, relatively closed, cup-shaped flowers with a round projection from the top side. Although some of the mutants in this study were not suitable for commercial cultivation, they could still be used for conventional crossings, to increase genetic variation and pass some of their traits along to hybrids with better combined phenotypes.

Overall, X-ray mutagenesis of Z. aethiopica seeds effectively expanded the range of traits we observed in Z. aethiopica plants and appears to have potential as an effective, time-saving method for enhancing genetic variability in this species. This is of particular interest for breeders working with the genus Zantedeschia, for which conventional breed- 
ing and interspecific crossings are limited by breeding barriers within the genus. The mutated lines are good candidates for commercialization and production as new Z. aethiopica cultivars suitable for cultivation under warm climate conditions.

\section{Conclusions}

$X$-ray radiation of Z. aethiopica seeds is an effective, feasible tool for increasing the phenotypic diversity of this ornamental plant. This approach allows the improvement of traits such as yield, early flowering under warm climate, appearance and resistance to devastating pathogens such as Pectobacterium. The results presented here demonstrate the potential of X-ray mutagenesis for future development of Z. aethiopica varieties, with traits such as better tolerance to viruses and different types of abiotic stress, improved scent and variation in spathe color.

Supplementary Materials: The following are available online at www.mdpi.com/xxx/s1, Figure S1: Calibration of the radiation dose for Zantedeschia aethiopica seeds, Figure S2: Flower diversity of mutated lines (CaX) during two growing seasons (2018-2020).

Author Contributions: Conceptualization, N. R. and I. Y.; Data curation, N. R. and B. S. S.; Funding acquisition, N. C. and I. Y.; Investigation, N. R., B. S. S., S. W., G. F. and M. S.-C.; Methodology, N. R., B. S. S., S. W., G. F. and M.I S.-C.; Project administration, N. R. and I. Y.; Resources, N. C. and I. Y.; Supervision, N. R. and I. Y.; Writing - original draft, N. R. and M. S.-C.; Writing - review \& editing, N. R.., N. C., I. Y. and M. S.-C. All authors have read and agreed to the published version of the manuscript.

Funding: This research was funded by Chief Scientist for Agriculture, Israel, grant number 20-010193.

Acknowledgments: We wish to acknowledge Yaron Cohavi and FloraHolland for providing the Zantedeschia trade report and allowing us to use the data in that report.

Conflicts of Interest: The authors declare no conflict of interest.

\section{References}

1. Hlophe, N.P., et al. Micropropagation of Zantedeschia aethiopica (L.) Spreng.: Towards its commercial use in the cut flower industry. Propagation of Ornamental Plants 2015, 15(2), 73-78.

2. Singh, Y.; vanWyk, A.E.; Baijnath, H. Floral biology of Zantedeschia aethiopica (L) Spreng (Araceae). South African Journal of Botany 1996, 62(3), 146-150.

3. Funnell, K.A. Zantedeschia, in The Physiology of Flower Bulbs; De Hertogh, A., Le Nard, M., Eds.; Elsvier Science Publisher: Amsterdam, Netherlands, 1993, pp. 683-704.

4. Letty, C. The genus Zantedeschia. Bothalia 1973, 11, 5-26.

5. Wei, Z., et al., Assessing genetic diversity and population differentiation of colored calla lily (Zantedeschia hybrid) for an efficient breeding program. Genes (Basel), 2017, 8(6),168.

6. Snijder, R.C., et al. Genetic variation in Zantedeschia spp. (Araceae) for resistance to soft rot caused by Erwinia carotovora subsp. carotovora. Euphytica 2004, 135(1), 119-128.

7. Snijder, R.C.; Lindhout, P.; van Tuyl, J.M. Genetic control of resistance to soft rot caused by Erwinia carotovora subsp. carotovora in Zantedeschia spp. (Araceae), section Aestivae. Euphytica 2004, 136(3), 319-325.

8. Guttman, Y., et al. Ecological adaptations influence the susceptibility of plants in the genus Zantedeschia to soft rot Pectobacterium spp. Horticulture Research 2021, 8(1), 13.

9. Yao, J.L.; Cohen, D. Multiple gene control of plastome-genome incompatibility and plastid DNA inheritance in interspecific hybrids of Zantedeschia. Theoretical and Applied Genetics 2000, 101(3), 400-406.

10. Yao, J.L.; Cohen, D.; Rowland, R.E. Plastid DNA inheritance and plastome-genome incompatibility in interspecific hybrids of Zantedeschia (Araceae). Theoretical and Applied Genetics 1994, 88(2), 255-260.

11. Yao, J.L.; Cohen, D.; Rowland, R.E. Interspecific albino and variegated hybrids in the genus Zantedeschia. Plant Science 1995, 109(2), 199-206.

12. Yedidia, I. Zantedeschia aethiopica. In Geophytes for Flowering in Israeli Agriculture: A Promise in the Ground. Ministry of Agriculture and Rural Development, Extension Services: Rishon LeZion, Israel, 2016, pp. $244-259$ (in Hebrew).

13. Ahloowalia, B.S.; Maluszynski, M. Induced mutations - A new paradigm in plant breeding. Euphytica 2001, 118(2), 167173.

14. Jain, S.M. Major mutation-assisted plant breeding programs supported by FAO/IAEA. Plant Cell Tissue and Organ Culture 2005, 82(1), 113-123. 
15. Jain, S.M. Mutagenesis in crop improvement under the climate change. Romanian Biotechnological Letters 2010, 15(2), 88106.

16. Kersey, C.M.; Agyemang, P.A.; Dumenyo, C.K. CorA, the magnesium/nickel/cobalt transporter, affects virulence and extracellular enzyme production in the soft rot pathogen Pectobacterium carotovorum. Molecular Plant Pathology 2012, 13(1), 58-71.

17. Ngamau, K. Selection for early flowering, temperature and salt tolerance of Zantedeschia aethiopica 'Green Goddess'. International Society for Horticultural Science (ISHS), Leuven, Belgium, 2008.

18. Ignatchenko, V., et al. VennDIS: A JavaFX-based Venn and Euler diagram software to generate publication quality figures. Proteomics 2015, 15(7), 1239-1244.

19. Yishay, M., et al. Differential pathogenicity and genetic diversity among Pectobacterium carotovorum ssp. carotovorum isolates from monocot and dicot hosts support early genomic divergence within this taxon. Environmental Microbiology 2008, 10(10), 2746-2759.

20. Schneider, C.A.; Rasband, W.S.; Eliceiri, K.W. NIH Image to ImageJ: 25 years of image analysis. Nature Methods 2012, 9(7), 671-675.

21. Holme, I. B., Gregersen, P. L., \& Brinch-Pedersen, H. (2019). Induced genetic variation in crop plants by random or targeted mutagenesis: convergence and differences. Frontiers in Plant Science, 10, 1468. Luzzatto, T., et al. Priming of antimicrobial phenolics during induced resistance response towards Pectobacterium carotovorum in the ornamental monocot calla lily. Journal of Agricultural and Food Chemistry 2007, 55(25), 10315-10322. Luzzatto, T., et al. Efficient, long-lasting resistance against the soft rot bacterium Pectobacterium carotovorum in calla lily provided by the plant activator methyl jasmonate. Plant Pathology 2007, 56(4), 692-701.

24. Luzzatto-Knaan, T., et al. Priming of protein expression in the defence response of Zantedeschia aethiopica to Pectobacterium carotovorum. Molecular Plant Pathology 2014, 15(4), 364-378.

25. Cho, H.R., et al. Development of two new Zantedeschia cultivars resistant to bacterial soft rot. Flower Research Journal 2014, 22(2), 88-94.

26. Cruz-Castillo, J.G.; Torres-Lima, P.A. 'Deja Vu' a new calla lily (Zantedeschia aethiopica) cultivar. Revista Chapingo Serie Horticultura 2017, 23(2), 97-101.

27. Tsror, L., et al. Characterization of Pectobacterium brasiliense strains from potato and vegetables in Israel. Plant Pathology 2021,. 00 1-9.

28. Maphosa, S.; Moleleki, L.N. Isolation and characterization of outer membrane vesicles of Pectobacterium brasiliense 1692. Microorganisms 2021, 9(9), 1918. 